de - im Ringen um eine gemeinsame Position - die Positionen der anderen Verbände kennen zu lernen und zu erörtern. Des Weiteren wurde die Beratungsvorlage zum vorbehaltlosen Grundeinkommen nicht angenommen - als zu unterschiedlich erwiesen sich die Positionen der Mitgliedsverbände.

Einig waren sich die Mitgliedsverbände, als es um eine Beratungsvorlage zu Frauen und Technik - „Ohne Frauen fehlt der Technik was“ - ging. Hier befürworteten alle Mitgliedsverbände, dass sich der Deutsche Frauenrat zukünftig stärker in die öffentliche Diskussion über Technikentwicklung, Technologiepolitik und Technikforschung einbringen soll.

Auf Antrag des djb wurde beschlossen, ein Fachgespräch zum Thema „Implementierung des Gender-Ansatzes in der Gesundheitsversorgung " durchzuführen, welches voraussichtlich am 12. Juni 2009 in Berlin stattfinden wird. Andere Beschlüsse betrafen ein Fachgespräch zur sexualisierten Gewalt in den Medien, die Forderung einer Geschlechterquotierung der Aufsichtsratsposten, die Begrenzung der Arbeitszeit bei geringfügigen Beschäftigungsverhältnissen auf maximal 50 Wochenstunden und die Forderung an den Gesetzgeber, $\mathbb{\int} 87$ Abs. 2 Nr. 1 AufenthaltsG dahingehend abzuändern, dass öffentliche Schulen nicht verpflichtet werden, die Ausländerbehörden zu unterrichten, wenn sie Kenntnis vom illegalen Aufenthalt einer Schülerin oder eines Schülers erlangen.

Fazit: Es wurde wieder ein bunter Strauß an Forderungen beschlossen. Nach nunmehr vierjähriger Delegiertentätigkeit bleibt festzustellen, dass die Arbeit des Deutschen Frauenrates auf all seinen vielfältigen Betätigungsfeldern nach meiner Einschätzung noch erfolgreicher umgesetzt werden könnte, wenn dieser bunte Strauß zu einem handhabbaren und überschaubaren Programm zusammengefügt wird. Dies fordert der djb seit längerem, weil die zahlreichen Beschlüsse für die Nutzerin im frauenpolitischen Alltag teilweise schon zu unübersichtlich geworden sind.

Aus djb-Sicht bedeutsam ist auch, dass eine „Arbeitsgruppe Entgeltgleichheit“ beschlossen wurde, in der ein Mitglied der djb-Kommission Arbeits-, Gleichstellungs- und Wirtschaftsrecht mitarbeiten wird. Sie soll nicht nur den „Equal Pay Day“ am 20. März 2009 vorbereiten, sondern auch die (2004 vom djb angestoßene) Debatte um geschlechtergerechte Tarifverträge neu beleben.

Und nun zu den Wahlen: Sie waren in diesem Jahr recht spannend, nachdem die ehemalige Vorsitzende Dr. Inge von Bönninghausen (Journalistinnenbund) als Gegenkandidatin zu der aus dem amtierenden Vorstand kandidierenden Marlies Brouwers (Arbeitsgemeinschaft katholischer Frauenverbände und -gruppen) angetreten war. Letztere wurde mit überzeugendem Vorsprung von 20 Stimmen gewählt, sie erhielt 66 der 112 abgegebenen Stimmen. Als Stellvertreterinnen wurden Claudia Menne vom DGB und Dr. Bettina Schleicher von Business and Professional Women gewählt. Unsere Kandidatin Almuth Kollmorgen wurde ebenfalls mit überzeugendem Ergebnis wiedergewählt. Weitere Beisitzerinnen sind Dr. Dagmar-E. Dennin vom Deutschen Ärztinnenbund, Dr. Kira Stein vom Deutschen Ingenieurinnenbund, Gabriele Wrede vom Deutschen Olympischen Sportbund, Ilona Helena Eisner von den Evangelischen Frauen in Deutschland und Sieglinde Scheel vom Demokratischen Frauenbund.

Dem neuen Vorstand ist zu wünschen, dass er die Vielfalt der Meinungen und Sichtweisen gut zusammenführt, auch wenn es hin und wieder gilt, intensive Diskussionsprozesse, gerade bei schwierigen Themen, durchzustehen. Im Vordergrund sollte immer der Nutzen der vielfältigen Expertise aus den unterschiedlichen Verbänden zum Wohle aller Frauen stehen.

\title{
Regionalgruppe München/Südbayern
}

\section{Forum Berufswege am 13. Oktober 2008}

Die Regionalgruppe München/Südbayern stellt in einem Forum „Berufswege im Gespräch“ in lockerer Folge interessante Juristinnen vor, um das Gespräch zwischen den Generationen anzuregen. Im Oktober 2008 war Hildegund Holzheid zu Gast, langjähriges Mitglied des djb und 1992 zur ersten Präsidentin eines Verfassungsgerichts in Deutschland gewählt.

Das Gespräch führte Sabine Lutzenberger. Anschließend gab es eine lebhafte Diskussion mit und unter den Zuhörerinnen.

\section{Frau Holzheid, wie sind Sie eigentlich zum Juristinnenbund gekommen?}

Es muss Anfang der 1970er Jahre gewesen sein, als mich Rechtsanwältin Marion Liebl-Blittersdorf einmal zu einem Juristinnen-Stammtisch eingeladen hat. Dort hat es mir sehr gut gefallen; die Kolleginnen waren mir sympathisch und erweiterten meinen juristischen Bekanntenkreis, vor allem um zahlreiche Rechtsanwältinnen. Ich bin wiedergekommen und wurde schließlich auch Mitglied des djb.

Mein frauenpolitisches Bewusstsein war sehr unterentwickelt. Das ist heute ganz anders. Zum Beispiel erscheinen mir Netzwerke unter Frauen wichtig, allein schon um sich auszutauschen, Informationen zu bekommen, sich gegenseitig zu bestärken und einander möglichst zu fördern. Männer tun das seit langem.

Als ich 1975 gemeinsam mit Marion Liebl-Blittersdorf eine Bundesvertreterversammlung des djb besuchte, wurden wir beide in den Bundesvorstand gewählt. In den Jahren 1975 bis 1977 war ich Mitglied im erweiterten Bundesvorstand, 1977 bis 1979 Schriftführerin. Nach zwei Amtsperioden wollten 


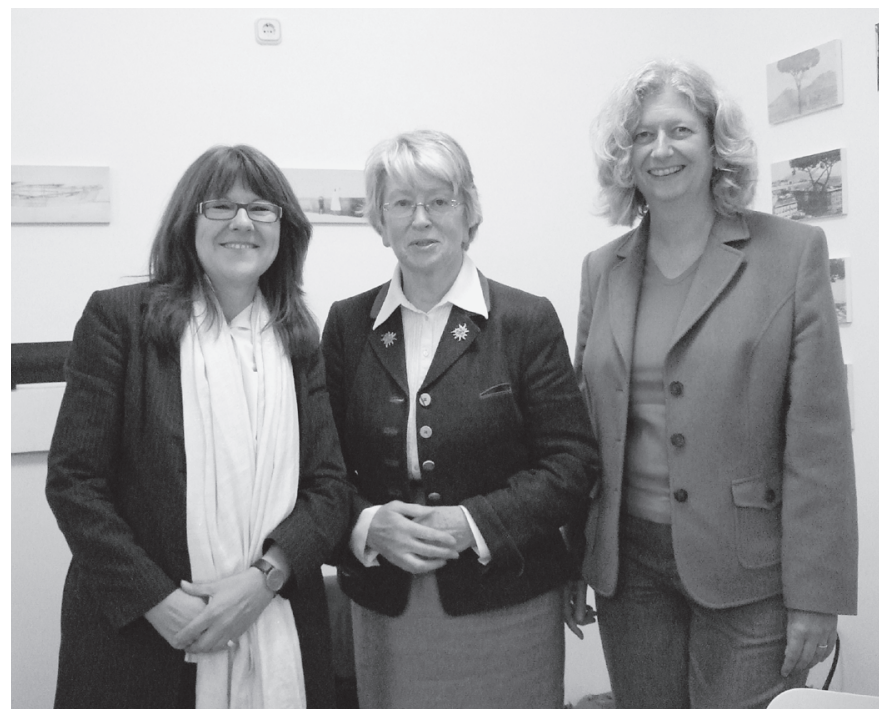

wir ausscheiden und haben zum Glück - für uns und für den djb - Gertrud Hofmann entdeckt, was - wie sich zeigen sollte - ein großer Gewinn für den djb war.

\section{Am Ende des Zweiten Weltkriegs waren Sie acht Jahre alt,} was hat Sie in Ihrer Entwicklung geprägt?

Ich hatte eine glückliche Kindheit und eine glückliche Jugend, auch wenn die Kriegs- und die Nachkriegsjahre viele Entbehrungen mit sich brachten. Aber es traf ja alle und wir konnten gut bei einem Glas Apfelsaft einen ganzen Abend fröhlich sein. Uns sind ja auch viele Gefährdungen erspart geblieben.

Ein Schulwechsel von Regensburg nach Nürnberg führte dazu, dass ich mich um ein Austauschschuljahr in den USA bewarb - erfolgreich bewarb. Das Schuljahr 1952/53 verbrachte ich im schönen Staat Pennsylvania und lebte dort in einer Familie, mit der ich heute noch Kontakt habe. Ein richtiger Austausch war das allerdings nicht. In meiner High School war ich „first German exchange student“, wurde überall herumgereicht, hatte keine Hemmungen, beim Rotary Club meines Geschichtslehrers einen Vortrag über „Deutschland“ zu halten, musste natürlich bei der Schulabschlussfeier eine Rede halten und bei der Schuloperette die Hexe singen und spielen. Es war eine wunderbare Zeit für mich und ich habe ein völlig anderes - partnerschaftliches - Verhältnis zwischen Lehrern und Schülern kennengelernt, als es damals bei uns üblich war. Auf dem Schiff, das mich nach Deutschland zurückbrachte, fand ich z.B. als Abschiedsgeschenk meiner Englischlehrerin „The complete works of William Shakespeare“ vor. Der Direktor meines deutschen Gymnasiums empfing mich dagegen mit großer Skepsis, ob ich wohl den Anschluss an meine alte Klasse schaffen würde. Das ist mir dann gut gelungen.

\section{Was hat Sie zum Jura-Studium bewogen?}

Eigentlich war mein bestes Schulfach Mathematik. Aber Lehrerin wollte ich nicht werden und was man alles sonst mit Mathe anfangen kann, habe ich erst viel später bei der TU München erfahren - als Kuratoriumsmitglied.
4 Rechtsanwältin Jutta Bartling, 1. Vorsitzende der Regionalgruppe München/Südbayern (links), Präsidentin des Bayerischen Verfassungsgerichtshofs und des Oberlandesgerichts München a. D. Hildegund Holzheid (Mitte), Schriftführerin Sabine Lutzenberger (rechts).

Foto: SABine FelLer

Nach meinen USA-Erfahrungen hatte ich die Vorstellung, ich müsste etwas Internationales, vielleicht etwas Völkerverständigendes machen. Ich dachte an Sprachen und Volkswirtschaft. Mein Vater, der selbst Richter war, mischte sich nicht ein, aber einer seiner besten Freunde, der selbst Volkswirt war, beklagte sein Schmalspurjuristendasein und riet mir dezidiert: „Kind, das machst du nicht. Studiere Jura; die Juristen haben immer die besseren und vielfältigeren Möglichkeiten. “ Das leuchtete mir ein.

\section{Wie war das Jura-Studium für Sie?}

Ich habe meine ersten zwei und die letzten drei Semester in Erlangen studiert und dazwischen zwei Bummelsemester in München gemacht. Frauen gab es nur wenige; die wenigen kannten einander und auch die Professoren kannten uns beim Namen, was dazu führte, dass wir immer aufgerufen wurden, um die unangenehmen Fragen zu beantworten.

Demokratie übten wir im Öffentlichen Recht bei Professor Maunz, der uns regelmäßig abstimmen ließ, wenn zwei Lösungen möglich erschienen. Dabei hat er es immer so hingedreht, dass die Mehrheit falsch lag und natürlich neugierig auf die richtige Lösung war. Diese Vorlesung fand immer zwischen elf und zwölf Uhr statt, was meinem etwas lockeren Tagesplan sehr entgegenkam.

Die Quittung kam im 5. Semester. Zurück in Erlangen erkannte ich - wie Faust - dass ich nichts wusste. Wahrscheinlich habe ich damit zum ersten Mal eine Ahnung bekommen, was juristisches Denken und Arbeiten heißt. Das habe ich mir schwer zu Herzen genommen und nach sieben Semestern dann das erste Staatsexamen gemacht.

\section{Wie sind Sie zur Justiz gekommen?}

In meiner Referendarzeit hat es mir besonders gut bei der $\mathrm{Zi}$ vilkammer am Landgericht Nürnberg-Fürth gefallen: die Richter waren relativ jung, modern und arbeiteten effektiv. Sie hatten auch so manchen flotten Spruch parat und waren sehr auf ihre richterliche Unabhängigkeit bedacht. Das hat mir ge- 
fallen. Dass ich meinem Ausbildungsrichter 40 Prozent seiner Urteile gemacht habe, hat mein Selbstbewusstsein sehr gestärkt. Ab da wusste ich, dass ich Richterin - Zivilrichterin - werden wollte. Als ich nach meinem zweiten Examen mein Interesse an einer Richterstelle in München kundtat, wurde mir zu meinem Schrecken - ich hatte die „Ministerialnote“ eine Stelle im Ministerium in Aussicht gestellt, was ich aber nicht wollte. So habe ich dann - wie in Bayern beim bewährten Laufbahnwechsel üblich - als Staatsanwältin angefangen.

Nach etwa drei Jahren wurde ich zur Richterin am Amtsgericht München ernannt. Als ich mich beim Amtsgerichtspräsidenten vorstellte und nach meiner zukünftigen Aufgabe fragte, meinte er: „Bisher haben wir die Frauen vor dem Erwachsenenstrafgericht ja verschont. Aber mit Ihnen wollen wir das Experiment einmal wagen. " Experiment - das war eigentlich eine Frauendiskriminierung, denn bei dem Mann, der mit der gleichen Justizbiografie zum Strafgericht kam, redete keiner von einem Experiment. Aber er hat es natürlich nicht diskriminierend gemeint, sondern wollte mir im Gegenteil sagen, dass er es mir zutraut, obwohl ich eine Frau bin.

Das waren eben die eingefahrenen Denkgleise.

\section{Wie erging es Ihnen bei der Justiz?}

Als Strafrichterin und Schöffengerichtsvorsitzende habe ich noch manchmal diese Pioniersituation zu spüren bekommen.

Sei es, dass die Schöffen nach dem „Herrn Rat“ fragten, wenn ich ins Beratungszimmer kam oder dass mich ein Zeuge einmal mit „Herr Vorsitzender“ anredete und zu dem in schallendes Gelächter ausbrechenden Staatsanwalt sagte: „Ich sehe schon, aber ich weiß nicht, wie man da sagt.“

Nachtrag: Solche Geschichten bleiben in Erinnerung, sollen aber den Blick auf den Ernst meiner Strafrichterzeit nicht verdecken. In Gewissensnöte bin ich als Strafrichterin fast nie gekommen, nicht beim Ausschöpfen des Strafrahmens nach oben und nach unten. Aber als einmal eine fahrlässig begangene Körperverletzung die Erblindung eines Arbeitskollegen des Angeklagten zur Folge hatte, habe ich lange mit mir gerungen.

Nach etwa zwei Jahren wurde ich noch einmal gefragt, ob ich ins Justizministerium kommen wolle und ich habe zugesagt. Ich arbeitete dort in der Strafrechtsabteilung, vorwiegend im Gnadenreferat, und in der Öffentlich-rechtlichen Abteilung, wo ich Stellungnahmen zu Verfassungsbeschwerden schrieb und u.a. Bundesratsangelegenheiten bearbeitete. Ich durfte damals auch an der Klage Bayerns gegen den Grundlagenvertrag mitwirken.

Im Jahr 1972 kam ich dann am Oberlandesgericht München endlich zu meiner ursprünglich angestrebten Tätigkeit, nämlich der einer Zivilrichterin.

Nach einigen Jahren hat man mir das Referat für Haushalts- und Bausachen in der Abteilung Strafvollzug im Justizministerium angeboten. Ich war etwas verblüfft, denn ich hatte keine Ahnung, kannte aber den Abteilungsleiter als eindrucksvollen und überaus sympathischen Mann und sagte ja.
Diesmal war ich es, die an ein Experiment dachte. Es wurden daraus zehn sehr gute Jahre, in denen es darum gegangen war, die Justizvollzugsanstalten auszubauen, gut auszustatten, Arbeits- und Ausbildungsstätten zu schaffen, mit den Kollegen im Finanzministerium zu verhandeln und unsere Anliegen im Haushaltsausschuss des Landtags zu vertreten.

Natürlich hatte es unter den - damals ausschließlich männlichen - Anstaltsleitern Vorbehalte gegeben, „ein solches Referat einer Frau“ anzuvertrauen. Als ob ich auf der Baustelle selbst hätte hinlangen müssen!

\section{Sie waren also mal wieder die erste Frau in einer Funktion.}

Ja, und das setzte sich noch fort. Und es hatte auch seinen Reiz.

Meine Erfahrung gerade im Strafvollzugsbereich ging dahin, dass frau dann, wenn sie einmal die erste Hürde genommen, die ersten Vorbehalte überwunden hat, es auch leichter haben kann und vielleicht sogar ein bisschen „verhätschelt“ wird. Da muss man sehr aufpassen, dass man dazu beiträgt, dass man nicht die einzige bleibt.

Ich bin natürlich in mancher Hinsicht überhaupt kein Vorbild für die jungen Kolleginnen: Ich war überhaupt nicht mobil und ich hatte keine Karriereziele. Ich war immer nur in München und wollte eigentlich am liebsten immer da bleiben, wo ich gerade war. Ich habe immer nur die Vorschläge bzw. Angebote der Justizpersonalverantwortlichen akzeptiert. Und damit bin ich gut gefahren. So wurde ich schließlich Leiterin der „Haushaltsabteilung“ im Justizministerium im Rang einer Ministerialdirigentin und mein damaliger Minister August Lang war sehr stolz darauf, die einzige Frau in diesem Rang in seinem Ministerium zu haben. Mentorinnen - nach solchen wurde ich oft gefragt - hatte ich bis dahin keine; es waren alles Männer, die die Entscheidungen getroffen hatten. Für mich war die Ministerialdirigentin eine herausragende Position, die ich natürlich für die Endstufe meiner Berufslaufbahn gehalten hatte.

Als mir ein damals schon längst pensionierter Generalstaatsanwalt einen Besuch abstattete und meinte, ich müsse jetzt schauen, wie es „weitergehe“, war ich eigentlich ziemlich empört, weil ich meine soeben erreichte Position zu gering gewürdigt sah und weil mir das Denken in Karriereleitern gar nicht lag.

\section{Die Macht haben Sie jedenfalls nicht gescheut?}

Wer etwas gestalten will, braucht die Macht dazu. Macht ist etwas anderes als Machtmissbrauch. Ich wünsche mir sehr, dass Frauen Macht wollen. Und mit dem Wort nicht so unendliche Schwierigkeiten haben.

\section{Was hat sich in Ihrer Karriere noch entwickelt?}

Zum 1. Juli 1992 ernannte mich die Bayerische Staatsregierung zur Präsidentin des Oberlandesgerichts München und der Bayerische Landtag wählte mich zur Präsidentin des Bayerischen Verfassungsgerichtshofs. Als Präsidentin eines Verfassungsgerichts war ich die erste in Deutschland, als Präsidentin 
eines Oberlandesgerichts die dritte. Der Vorschlag kam von der damaligen bayerischen Justizministerin Dr. Mathilde Berghofer-Weichner, die leider vor einigen Monaten verstorben ist. Sie war ein treues Mitglied des djb, eine starke Frau und mir eine gute Freundin.

Dass diese beiden Ämter für eine Juristin, die einfach nur Richterin werden wollte, eine Krönung ihres Berufslebens waren, ist klar.

Die Bayerische Verfassung hat ja die (Landes-)Verfassungsgerichtsbarkeit sehr stark ausgebaut, insbesondere mit Verfassungsbeschwerden, Organstreitigkeiten, Popularklagen, einer Verfahrensart, die es nur in Bayern gibt und bei der jeder Bürger die Verfassungswidrigkeit einer Norm rügen kann, auch wenn er nicht in eigenen Rechten verletzt ist. Auch zahlreiche Entscheidungen über die Zulässigkeit von Volksbegehren sind in meiner Amtszeit angefallen. Mit zwischen 100 und 150 Verfahren pro Jahr waren wir gut beschäftigt; und es gibt zwar zahlreiche Spruchgruppen beim VerfGH, aber die Präsidentin war in jeder die Vorsitzende. Die Rechtsprechungstätigkeit am Verfassungsgericht machte einen großen Teil meines Gesamtarbeitspensums aus. Und als Präsidentin des Oberlandesgerichts waren es die Justizverwaltungsaufgaben - wie Personal, Haushalt, Bau, EDV, Referendare, Geschäftsprüfungen etc. in einem der größten OLG-Bezirke Deutschlands, die mich forderten, mir aber auch viel Freude machten.

Ich habe mich sehr bemüht, Frauen zu fördern und in Führungspositionen zu bringen, d.h. zunächst einmal Frauen für solche Positionen zu gewinnen. Das war nicht immer einfach, weil sich manchmal besonders tüchtige Frauen auch verweigern. Wenn Grund dafür die schwierige Vereinbarkeit von Beruf und Familie war, habe ich auch volles Verständnis dafür. Es gab aber auch Fälle, die ich nicht verstehen konnte. War es die Scheu vor der vermuteten Einsamkeit in der Führungsrolle? War es Angst davor, höher zu steigen als der Ehemann?

Immer wieder habe ich erlebt, dass Frauen auf ein Angebot meinerseits hin fragten: „Ja, meinen Sie, dass ich das schaffe? “, dann aber doch gerne zugriffen.

Es ist mir jedenfalls gelungen, eine ganze Reihe von Frauen in die Positionen zu bringen, auf deren Besetzung ich maßgeblichen Einfluss hatte: Referentinnen in der Verwaltung des OLG, Amtsgerichtsdirektorinnen, Richterinnen am Verfassungsgerichtshof, eine Referentin des Verfassungsgerichtshofs, die mittlerweile dessen Generalsekretärin ist, und last not least eine Frau als meine Nachfolgerin und eine als Präsidentin des größten bayerischen Landgerichts München I.

\section{Haben Sie selbst einmal renommierte Angebote abgelehnt?}

Ja, zum einen das Angebot, in Bayern Justizministerin zu werden, und zum anderen die Anfrage, zum Bundesverfassungsgericht nach Karlsruhe zu gehen.

Ich kann so offen darüber reden, weil beides seinerzeit durch die Zeitungen gegangen war und Ministerpräsident Stoiber sogar im Bayerischen Fernsehen ganz offen darüber gesprochen hat, dass er mich als Ministerin gewinnen wollte. Ich wollte allerdings nicht in die Politik gehen.

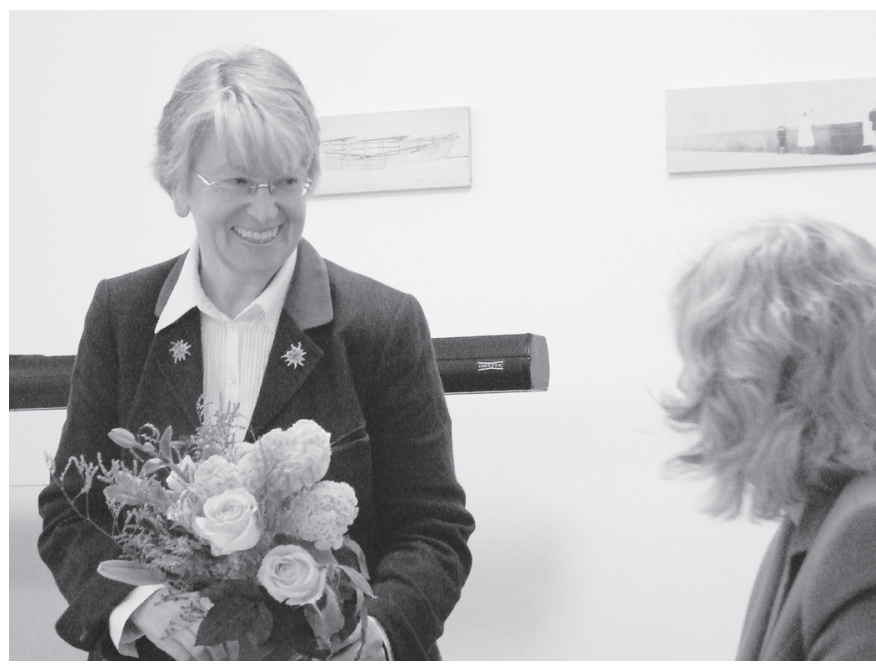

$\triangle$ Präsidentin des Bayerischen Verfassungsgerichtshofs und des Oberlandesgerichts München a. D. Hildegund Holzheid nach dem Gespräch.

Foto: SABine Feller

Das Wort Ruhestand müsste man bei Ihnen eher in Anführungszeichen setzen, wenn ich mir anschaue, wo Sie überall ehrenamtlich tätig sind. Sie sind Vizevorsitzende der Bioethikkommission Bayern, Mitglied im Verwaltungsrat des ZDF, Vorsitzende des Kuratoriums der Technischen Universität München, Mitglied in den Kuratorien der Evangelischen Akademie Tutzing und der Eugen-Biser-Stiftung. Und 2008 wurden Sie vom Präsidenten des Deutschen Bundestags in den Deutschen Ethikrat berufen. Worum geht es da eigentlich?

Der Deutsche Ethikrat ist Nachfolger des Nationalen Ethikrats, aber auf gesetzlicher Grundlage errichtet und nicht nur von der Bundesregierung bestellt, sondern vom Deutschen Bundestag, wobei Bundestag und Bundesregierung je 13 Mitglieder vorgeschlagen haben. Gegenstand unserer Arbeit sind die Fragen, die sich im Zusammenhang mit der Forschung und den Entwicklungen insbesondere auf dem Gebiet der Lebenswissenschaften und ihrer Anwendung auf den Menschen ergeben. Zu unseren Aufgaben gehören die Erarbeitung von Stellungnahmen und Empfehlungen für politisches und gesetzgeberisches Handeln und die Information der Öffentlichkeit. Dabei soll die breit gestreute Zusammensetzung des Rates dazu dienen, dass die schwierigen ethischen Fragen durch ein breit gefächertes Meinungsspektrum und durch Beteiligung aller in Frage kommender Fachgebiete eingehend aufbereitet werden, um damit Grundlage für politische Entscheidungen sein zu können. Themen sind z.B. Biobanken, anonyme Geburt, Mangel im Überfluss (Ernährung), Hirnforschung. Entscheiden ist nicht unsere Aufgabe; das ist Aufgabe der Politik.

In der Bayerischen Bioethikkommission, der ich seit 2001 angehöre, haben wir uns u.a. mit dem Import humaner embryonaler Stammzellen, mit der Präimplantationsdiagnostik, mit der Patientenverfügung, der Sterbehilfe und dem Schutz des behinderten ungeborenen Lebens befasst. 
Fragen aus dem Kreis der Zuhörerinnen:

\section{Wie kommt es, dass Sie keine Kinder haben?}

Ich hatte einfach nicht zur richtigen Zeit den richtigen Partner. Als ich den gefunden hatte, war ich für Kinder schon zu alt.

Ich sage aber auch, dass den Frauen lange Zeit die Vereinbarung von Familie und Beruf sehr schwer gemacht wurde. Umso lebhafter unterstütze ich den Ausbau von Kinderbetreuungseinrichtungen und Ganztagsschulen. Ich finde es für Kinder auch gut, wenn sie möglichst bald auch außerhalb der Familie Anregungen bekommen, besonders wenn es sich um Einzelkinder handelt.

Ich freue mich übrigens sehr, dass in Bayern so viele Väter die Elternmonate in Anspruch nehmen.

Meinen Sie, dass Ihr Berufsweg von männlichen Kollegen auch so akzeptiert worden wäre, wenn Sie Kinder hätten?

Daran habe ich keinen Zweifel. Schauen Sie sich meine Nachfolgerin Edda Huther an; sie hat es mit drei Kindern geschafft. Natürlich mit Hilfen und einer Tagesmutter, aber sehr imponierend ohne Beurlaubung. Die Frage ist wohl nicht, ob die männlichen Kollegen das akzeptieren, sondern, ob man es selbst schafft.

Ich gehe übrigens hoffnungsvoll davon aus, dass man auf Dauer auf die Frauen und ihre Fähigkeiten gar nicht mehr verzichten kann. Dafür spricht schon die demografische Entwicklung. Und offen gesagt: es ist mir lieber, gebraucht zu werden als nur geduldet.
Wir Frauen haben auch ein Defizit darin, Netzwerke zu bilden. Bundeskanzlerin Angela Merkel hat dazu beim 20-jährigen Jubiläum des Bundesfrauenministeriums sehr treffend gesagt, Frauen müssten viel mehr Bündnisse schmieden. „Männer können sich auch nicht immer alle leiden. Aber wenn es um Posten geht, arbeiten sie bestens zusammen.“

Was raten Sie jüngeren Kolleginnen, um im Beruf voranzukommen?

Sie sollten das machen, was sie wirklich gerne machen. Nur dann kann man auch gut sein.

Ob ich auf meinem Weg auch Ellenbogen gebraucht hätte, wurde ich vorhin gefragt. Eindeutig nein; in der bayerischen Justiz wäre das sicher schädlich gewesen.

Ich bin gut damit gefahren, immer das anzunehmen, was mir die Personalverantwortlichen in der Justiz angeboten haben. Natürlich schadet es nicht, wenn man auffällt - positiv auffällt, z.B. durch Übernahme von Spezialaufgaben.

Echtes Engagement für die eigenen Aufgaben ist wichtig und die Fähigkeit und die Bereitschaft zur Kommunikation.

Vielen Dank für das Gespräch und Ihre lebhaften Schilderungen, Frau Holzheid. Wir nehmen viele Denkanstöße mit und freuen uns sehr, Sie in unserer Regionalgruppe zu haben. Sie sind dem Juristinnenbund über viele bewegte Zeiten treu geblieben, auch wenn Sie manchmal anderer Meinung waren. Das verdient besondere Anerkennung.

\section{Besuch im Pressehaus der Nordwest-Zeitung (NWZ)}

\section{am 7. Januar 2009}

Über die Informationsverarbeitung und Produktionsabläufe in dem Medienunternehmen informierten Gaby Schneider-Schelling, Chefin vom Dienst und Sabine Schicke, stellv. Leiterin der Lokalredaktion.

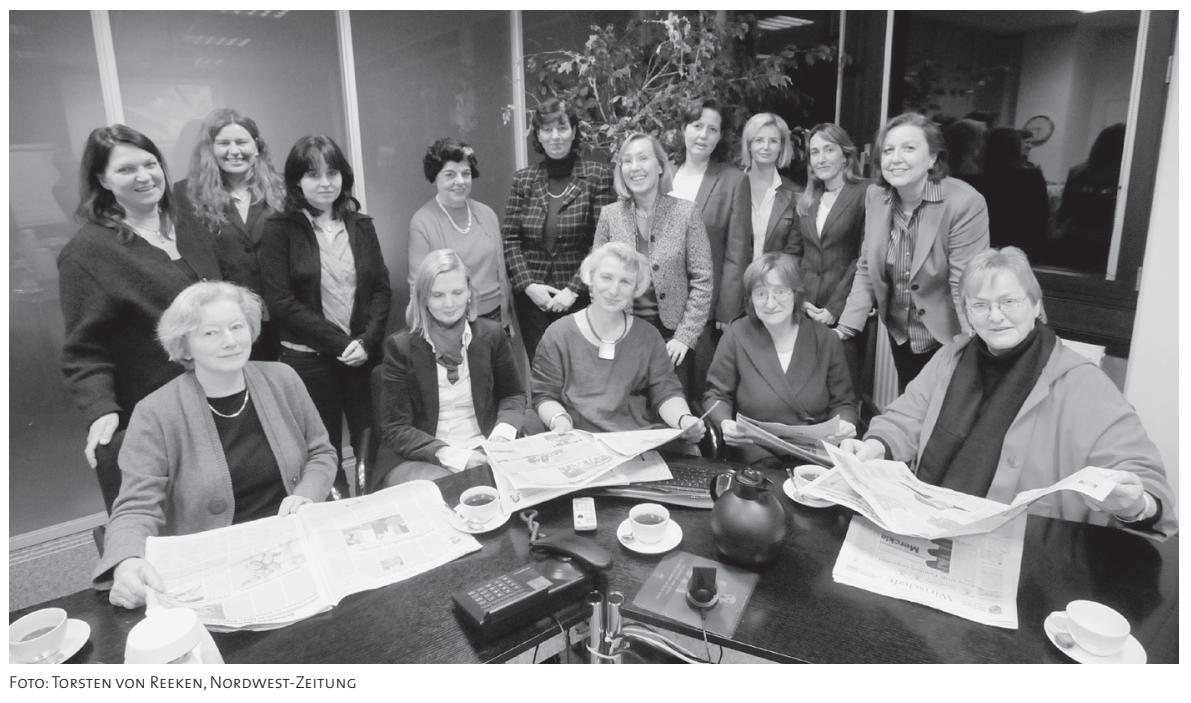

4 V.I.n.r. oben: Gaby Schneider-Schelling, NWZ, Chefin vom Dienst; Imke Janssen, Dipl.Kffr.; Carola Riekens, Verwaltungsangestellte; Margretlies Neumann-Nieschlag, Rechtsanwältin; Birgit Kersten, Rechtsanwältin und vereidigte Buchprüferin; Ingrid Spalthoff, Richterin am LG; Inge Saathoff, Rechtsanwältin; Kerstin Jansen, Rechtsanwältin; Raquel Cerezo-Ramirez, Abogada; Sabine Schicke, NWZ Stellv. Leiterin der Lokalredaktion Oldenburg.

$\varangle$ V.I.n.r. unten: Dagmar Weber, Vors. Richterin am OLG i.R.; Maike Bartlmae, Rechtsanwältin; Micaela Schweers-Sander, Verwaltungsoberrätin; Dr. Bettina Graue, Vertretungsprofessorin; Inge Böttcher, Rechtsanwältin. 